\title{
A Review of Management of First-Time Dislocations in Contact Sport Athletes
}

\author{
R Brankin* and J Sinha \\ Rory Brankin, King's College Hospital, London, UK \\ Received: 觜 September 17, 2018 Published: 制 September 24, 2018 \\ *Corresponding author: Rory Brankin, King's College Hospital, London, UK
}

Abstract

A review of the current management concept in treating first time dislocations in contact sport athletes. Non-operative management provides the fastest return to play but the redislocation rates in contact athletes are extremely high. Latarjet stabilization at the earliest practical opportunity provides the contact athlete with the best chance of returning to competition and staying there.

\section{Introduction}

Shoulder dislocations and instability events are a common occurrence in contact sport athletes (2.58 dislocations per 100,000 Athletic Exposures) [1]. The most common cause of dislocation is tackling [2].

\section{Discussion}

In the past the management of first-time shoulder dislocations was immobilization and then rehab. This had a high recurrence rate and, due to the prolonged immobilization, a slow return to sporting activities. A lack of evidence for any particular immobilization protocol has led to the widespread adoption of early mobilization and rehabilitation. This avoids the stiffness and allows for rapid return to play (3 weeks for NFL players) [3] but does not address the high recurrence rate (55\% for NFL players) [3]. Recurrent dislocations are problematic for several reasons, not just that each episode removes the athlete from play for a period of time. Each dislocation is likely to increase the bony defects associated with the injury on both the glenoid (Bony Bankart) and the humeral head (Hill-Sachs) and the outcomes of stabilization surgery deteriorate as the size of the bony defect increases. Each episode of the shoulder moving beyond its normal anatomical range of motion and position also exposes the athlete to a risk of neurological compromise, which is likely to be more career limiting than shoulder instability itself. Axillary nerve injury was present in $15.5 \%$ of traumatic anterior dislocations on nerve conduction studies [4].

Increasingly surgical stabilization is offered to contact sport athletes after a single instability episode with the timing dependent on where the injury occurred in the playing season. An NFL player injured at the start of the season has a very high chance of reinjury before reaching a time when surgery will not require him to miss more play than a player injured with only 8 weeks before the off season. Surgical stabilization procedures fall into 4 main categories: Arthroscopic Bankart repair, open stabilization, Bristow-Latarjet or Eden Hybinette type procedures. The latter two can be performed open or arthroscopically. Open Stabilization has a relatively low (66\%) [5] slow (8.2 months) [6] rate of return to play and a fractionally higher recurrence rate (6.7\%) [5]. Arthroscopic Bankart repair has a high rate of return to previous activity levels (71\%) [5] and a low time to return to sport (5.9 months) [6] but a higher rate of recurrence than open Latarjet (6.6\%) [5]. This effect becomes more pronounced as the amount of anterior glenoid bone loss increases (to the extent that Arthroscopic Bankart is not appropriate in contact athletes with any significant anterior glenoid bone loss). Traditional Eden-Hybinette open procedures have been associated low redislocation rates (4.8\%) [7] but a high rate of glenohumeral arthritis (80\%) [7].

Open Bristow-Latarjet procedures have been controversial in the past as they are non anatomic and require injury to the subscapularis muscle but have the best results for returning to pre injury activity levels (73\%) [5] and the best redislocation rates (3.5\%) [5] and the lowest time to return to sport (5.07 months) [6]. Arthroscopic Latarjet procedures are not as predictable in terms of outcome as open Latarjets but this may be something that improves with numbers as it is a technically very challenging intervention. The future has interesting possibilities for arthroscopic modifications of the Eden-Hybinette as combining a labral repair with an extra- 
articular bone block may provide the improved bony stability without the accelerated wear of the traditional operation.

\section{Conclusion}

Contact sport athletes with first time shoulder dislocations should be considered for surgical stabilization at the earliest convenient time in their schedule. The Bristow-Latarjet stabilization is the most predictable intervention for getting them back to full contact and keeping them there. An arthroscopic Bankart repair is similar in terms of outcome and has the advantage of allowing revision to Latarjet if it fails.

\section{References}

1. Kraeutler MJ, Currie DW, Kerr ZY, Roos KG, McCarty EC, et al. (2018) Epidemiology of Shoulder Dislocations in High School and Collegiate Athletics in the United States: 2004/2005 Through 2013/2014. Sports Health 10(1): 85-91.
2. Bonacci J, Manson B, Bowe SJ, Gill S, Seward H, et al. (2018) Operative shoulder instability injury management in Australian Football League players: A case series. J Sci Med Sport 21(8): 760-764.

3. Okoroha KR, Taylor KA, Marshall NE, Keller RA, Fidai M, Mahan MC, Varma V, Moutzouros V (2018) Return to play after shoulder instability in National Football League athletes. J Shoulder Elbow Surg 27(1):17-22.

4. Atef A, El Tantawy A, Gad H, Hefeda M (2016) Prevalence of associated injuries after anterior shoulder dislocation: a prospective study. Int Orthop 40(3): 519-24.

5. Ialenti MN, Mulvihill JD, Feinstein M, Zhang AL, Feeley BT (2017) Return to Play Following Shoulder Stabilization: A Systematic Review and Metaanalysis. Orthop J Sports Med 5(9): 2325967117726055.

6. Abdul Rassoul H, Galvin JW, Curry EJ, Simon J, Li X (2018) Return to Sport After Surgical Treatment for Anterior Shoulder Instability: A Systematic Review. Am J Sports Med 1:363546518780934.

7. Jerosch J, Schröder M, Steinbeck J, Voss M (1995) Long-term results of the modified Eden Hybinette operation for treatment of recurrent shoulder dislocation. Un fall chirurg 98(1):13-20.

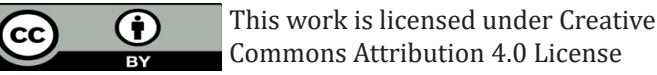

To Submit Your Article Click Here:

Submit Article

$\begin{gathered}\text { Orthopedics and Sports Medicine } \\ \text { Open Access Journal }\end{gathered}$
Assets of Publishing with us
- Global archiving of articles
- Immediate, unrestricted online access
- Rigorous Peer Review Process
- Authors Retain Copyrights
- Unique DoI for all articles

